\title{
The effect of Ginkgo biloba against ferric nitrilotriacetate and/or low doses of gamma irradiation induced renal injury in rats
}

\author{
Mohamed R. Mohamed ${ }^{1}$, Neamat Hanafi Ahmed Osman ${ }^{2 *}$, Somia Zakria ${ }^{2}$ and Marwa El-Helw ${ }^{2}$ \\ ${ }^{1}$ Biochemistry Department, Faculty of Science, Ain Shams University, Cairo, Egypt \\ ${ }^{2}$ National Center for Radiation Research and Technology, Atomic Energy Authority (AEA), Cairo, Egypt
}

A R T I C L E I N F O

Article history:

Received 28 July 2016

Accepted 21 November 2016

Keywords:

Ginkgo biloba;

Inflammation;

Oxidative stress;

Renal carcinogenesis;

Caspase 3.

\begin{abstract}
A B S T R A C T
The present study was designed to investigate the efficacy of Ginkgo biloba (GB) and low doses of gamma irradiation against ferric nitrilotriacetate (Fe-NTA) promote renal carcinogenesis in Wister rats. Renal carcinogenesis was initiated by single intraperitoneal injection of $\mathrm{N}$-nitrosodiethylamine (DEN $200 \mathrm{mg} / \mathrm{kg}$ body weight) and promoted by twice weekly administration of Fe-NTA $9 \mathrm{mg}$ $\mathrm{Fe} / \mathrm{kg}$ body weight for 16 weeks. Animals (whole body) were exposed to $50 \mathrm{cGy}$ per week for 19 weeks at a total dose of $9.5 \mathrm{~Gy}$. The efficacy of GB was studied in terms of lipid peroxidation (LPO), renal anti-oxidant armory such as catalase, superoxide dismutase, glutathione peroxidase and glutathione (GSH) and plasma toxicity markers. Also, caspase 3 and renal Histopathological changes were detected. Administration of Fe-NTA and exposure to low doses of $\gamma$-radiation enhance renal LPx, with concomitant reduction in reduced GSH content and antioxidant enzymes. It induces serum toxicity markers, viz., blood urea nitrogen and creatinine. Histopathological alterations and renal caspase 3 enhancement were detected in kidney tissue. Chemo preventive effects of GB were associated with upregulation of antioxidant armory and down regulation of plasma toxicity markers. GB was also able to down regulate of caspase 3. Histopathological changes further confirmed the biochemical results. In conclusion: These results provide a powerful evidence for the preventive efficacy of GB against renal carcinogenesis induced by either low doses of $\gamma$ - irradiation or Fe-NTA.
\end{abstract}

\section{Introduction}

Epidemiological studies have shown that increased body iron storage is associated with an increased risk of cancer and early death. Suzy and Frank ${ }^{[1]}$ reported that iron can contribute to tumour initiation, tumour growth, tumour microenvironment and in metastasis. Mechanisms whereby iron may act in carcinogenesis are induction of oxidative stress, facilitation of tumor growth and modification of the immune system. Metal ions react with superoxide anion $\left(\mathrm{O}^{-}{ }^{2}\right)$ and $\left(\mathrm{H}_{2} \mathrm{O}_{2}\right)$ to produce highly reactive species such as hydroxyl free radical $(\mathrm{OH})$ and metal-oxygen complexes in biological systems, resulting in oxidative DNA damage. $\mathrm{H}_{2} \mathrm{O}_{2}$ induced oxidative DNA damage in cells has been thought to result from the formation of hydroxyl free radicals through Fenton reaction with iron ${ }^{[2]}$.

The development of renal carcinogenesis has been linked with innumerable risk factors including environmental exposure to different toxicants ${ }^{[3]}$.

\footnotetext{
* Corresponding author.

E-mail address: neamatosman@yahoo.com
}

Nitrilotriacetic acid (NTA) is an aminotricarboxylic acid with an empirical formula of $\mathrm{C}_{6} \mathrm{H}_{9} \mathrm{NO}_{6}$. NTA can make complexes with metal ions such as $\mathrm{Fe}^{3+}$ or $\mathrm{Cu}^{2+} \mathrm{Fe}-\mathrm{NTA}$, a complex of $\mathrm{Fe}^{3+}$ and NTA, is a strong nephrotoxic agent and a renal carcinogen. It is an established fact that an iron-chelate of nitrilotriacetate, ferric nitrilotriacetate (Fe-NTA) induces acute and sub-acute renal injury in animals ${ }^{[4-6]}$. It is evidenced from previous reports that oxygen free radical was formed from redox-active iron and was detected in the serum of Fe-NTA-treated rats $[7,8]$

Medicinal plants have been used by all civilization as a basis of medicines since ancient times. In the recent times natural products have been used to prevent the toxicities induced by chemicals, drugs and carcinogenic xenobiotics. Plant based products are generally considered safe and proved to be effective against various human ailments and their medicinal uses have been gradually increasing in developed countries. Epidemiological studies have also proven that consumption of vegetables and fruit have caused lower 
incidence of cancers ${ }^{[9]}$. Interest in medicinal plants as a re-emerging health aid also has been encouraged by the rising costs of prescription drugs in the maintenance of personal health and wellbeing, and the bioprospecting of new plant derived drugs.

Ginkgo biloba, a member of the family Ginkgoaceae, was cultivated in China in the mid1700s. It is used medically today as a standardized preparation which contains $240 \mathrm{mg} / \mathrm{g}$ flavonoids (ginkgoflavone glycosides) and $60 \mathrm{mg} / \mathrm{g}$ terpenoids (ginkgolides and bilobalides) which are the most important active ingredients in the extract. The flavonoids act as free radical scavengers, especially for oxygen-derived free radicals, and to neutralize ferryl ion-induced peroxidation ${ }^{[10]}$. The terpenoids are known as antagonists of platelet-activating factor, which implicates in the processes of platelet aggregation and arterial thrombosis, acute inflammation, allergic reactions and cardiovascular insufficiency ${ }^{[11,12]}$. Ginkgo biloba is used to improve cardiovascular circulation and to lessen cerebrovascular insufficiency in western countries clinically ${ }^{[13]}$.

Radiation hazards present an enormous challenge for the biological and medical safety. The deleterious effects of ionizing radiation in biological systems are mainly mediated through the generation of reactive oxygen species (ROS) in cells as a result of water radiolysis ${ }^{[14]}$. ROS and oxidative stress may contribute to cytotoxicity and to metabolic and morphologic changes in animals and humans ${ }^{[15]}$. Nevertheless, the possibility that low doses of radiation may have beneficial effects has been the subject of considerable debate ${ }^{[16]}$. Evidences accumulated over the past decade have indicated that living organisms, including humans, respond differently to low dose/low dose-rate radiation than they do to high dose/high dose-rate radiation ${ }^{[17]}$. The radioadaptive response, radiation-induced bystander effects, low-dose radio-hypersensitivity, and genomic instability are specifically observed in response to low dose/low doserate radiation, and the mechanisms underlying these responses often involve biochemical/molecular signals that respond to targeted and non-targeted events ${ }^{[17]}$.

The objective of this work is to evaluate the role of Ginkgo biloba against ferric nitrilotriacetate and/or low doses of gamma irradiation induced renal carcinogenesis.

\section{Materials and Methods}

\section{Chemicals}

All chemicals and reagents used were of the highest purity available and purchased from either Merck (Darmastadt, Germany), or sigma Aldrich Chemie (Deisenhofen, Germany). Kits for BUN and creatinine were supplied by Diamond Diagnostics, Egypt. Kit for Caspase 3 was supplied by Enzo life sciences, New York, USA.

\section{Preparation of Fe-NTA solution}

Fe-NTA is prepared fresh immediately before its use as per Awai et al. ${ }^{[4]}$. To prepare Fe-NTA, ferric nitrate solution is mixed with fourfold molar excess of disodium salt of NTA and the pH is adjusted to 7.4 with sodium bicarbonate solution. The dose of NTA is $9 \mathrm{mg} \mathrm{Fe} / \mathrm{kg}$ body weight twice a week for 20 weeks.

\section{Radiation process}

Animals were exposed to whole body gamma radiation using a Canadian gamma cell-40, $\left({ }^{137} \mathrm{Cs}\right)$ at the National Center for Radiation Research and Technology (NCCRT), Cairo, Egypt. The irradiation dose rate was $0.61 \mathrm{~Gy} / \mathrm{min}$. Animals (whole body) were exposed to50 cGy per week for 19 weeks at a total dose of $9.5 \mathrm{~Gy}$.

\section{Ginkgo biloba treatment}

Ginkgo biloba extract was provided by Changsha Sunfull Biotech Co., Ltd., China, was dissolved in saline then orally administered to rats with dose of $100 \mathrm{mg} / \mathrm{kg} / \mathrm{day}$ [18], 3 times per week for 21 weeks starting from one week before Fe-NTA administration.

\section{Experimental animals}

Eighty adult Male albino rats (120-150 g) obtained from Al-Neil Company for drugs; Cairo, Egypt. Animals were housed in cages under good ventilation condition. The Animals were kept in isolated cages, under standard laboratory conditions including all hygienic measures with constant illumination, temperature and humidity.

\section{Experimental design:}

The animals were allowed 7days for adaptation. The experimental animals were divided into eight groups $(\mathrm{n}=$ 10), namely (1) Control healthy animals received only saline injection intraperitoneally $(0.85 \% \mathrm{NaCl})$ at a dose of $10 \mathrm{ml} / \mathrm{kg}$ body weight; (2) (GB): Rats received Ginkgo biloba extract; (3) (IR): Animals were exposed to $\gamma$-radiation; (4)(Fe-NTA): Animals were initiated with a single i.p. injection of DEN at a dose level of 200 $\mathrm{mg} / \mathrm{kg}$ body weight in saline. Ten days after initiation, the animals were promoted with intraperitoneal injection of Fe-NTA at a dose level of $9 \mathrm{mg} \mathrm{Fe} / \mathrm{kg}$ body weight, twice a week for 20 weeks; (5) (GB + IR): Animals received Ginkgo biloba extract and were exposed to $\gamma$ irradiation; (6) (GB +Fe-NTA): Animals received Ginkgo biloba extract and were injected Fe-NTA; (7) (Fe-NTA +IR): Animals were injected Fe-NTA and exposed to $\gamma$-radiation and (8) $(\mathbf{G B}+\mathbf{F e}-\mathbf{N T A}+\mathbf{I R})$ : Animals received Ginkgo biloba extract and were injected Fe-NTA and exposed to $\gamma$-radiation.

\section{Samples:}

At the end of the experimental period (21 weeks) blood samples were taken from rats under diethyl ether anesthesia by heart puncture. Blood samples were collected in heparinized tubes, centrifuged at 3000 r.p.m. for 10 minutes. The whole blood was used for determination of antioxidant markers. Whereas, plasma was separated in vials for determination of other parameters. The kidneys of experimental animals were dissected out and divided into two parts, one part was kept in 10\% formalin for histopathological studies and the other part was homogenized in $(10 \% \mathrm{w} / \mathrm{v})$ phosphatebuffered-saline $(0.02 \mathrm{M}$ sodium phosphate buffer with $0.15 \mathrm{M}$ sodium chloride, $\mathrm{pH}$ 7.4). 


\section{Biochemical determinations}

For antioxidant markers, glutathione (GSH) content was determined according the method of Beutler et al. ${ }^{[19]}$, glutathione peroxidase (GPX) activity level was evaluated according method mentioned by Gross et al. [20], superoxide dismutase (SOD) and Catalase (CAT) activities were evaluated according to the method of Minami andYoshikawa ${ }^{[21]}$ and Sinha ${ }^{[22]}$, respectively. The estimation of lipid peroxidation (LPx) was done following the method described by Yoshioka et al. ${ }^{\text {[23] }}$ and nitric oxide (NO) was determined according to Miranda et al ${ }^{[24]}$. Kidney tissue homogenates of each group was assayed for caspase-3 activity using rat caspase-3 ELISA kit.

\section{Statistical analysis:}

The SPSS (version 10) was used in data analysis. Data were analyzed with one-way analysis of variance (ANOVA) followed by a post hoc test (LSD alpha) for multiple comparisons. The data were expressed as mean \pm standard error $(\mathrm{SE})$. $\mathrm{P}$ values $<0.05$ were considered to be statistically significant.

\section{Results}

Effect of Ginkgo biloba on kidney caspase-3 activity A significant decrease in activities of kidney caspase 3 observed in group subjected to Fe-NTA compared with the negative control group. Prophylactic and therapeutic treatment with GB significantly increases caspase3 activity (Fig. 1).
The levels of plasma creatinine and BUN were significantly increased after exposure to low doses of $\gamma$ radiation and Fe-NTA treatment either alone or combined compared with control group level (Table 1). Prophylactic treatment with GB significantly reduced the elevation in the plasma creatinine level compared with the previous treatments. In kidney tissue, a significant increase in MDA level was observed in $\gamma$-irradiated rats compared to control rats (Table 1). Also, the same observation was recorded after Fe-NTA treatment either alone or combined with $\gamma$-irradiated compared to control level. Prophylactic treatment with GB for 21 weeks showed a significant protection against Fe-NTA or $\gamma$ irradiation induced lipid peroxidation in kidney and offered a significant decrease in MDA level compared to $\gamma$-irradiated rats. Reverse directed observations were detected in NO levels when Fe-NTA or $\gamma$-irradiated rats treated with GB for 21 weeks either alone or in combined.

Treatment of the experimental animal with G. biloba represents a non significant change in Kidney antioxidants markers (GSH, CAT, GSH-px and SOD) in Table 2. Whereas either $\gamma$ - irradiation or Fe-NTA treatments led to a significant depletion in kidney antioxidants markers (GSH, CAT, GSH-px and SOD) when compared with their equivalent values in control rats. Pre-treatment with GB improved significantly kidney antioxidants markers compared to the Fe-NTA group (Table 2).

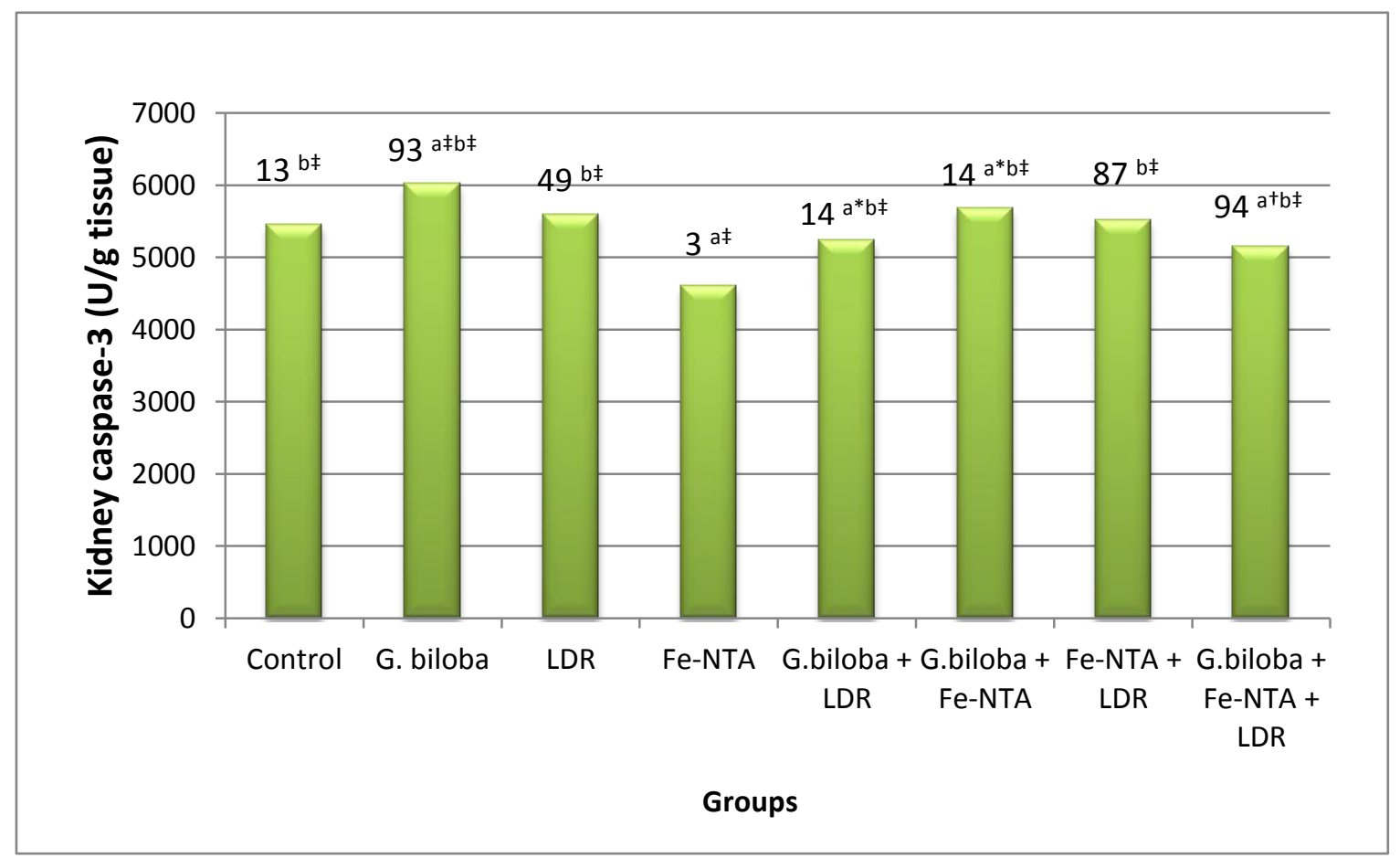

Fig. (1): Kidney caspase-3 activity. a: significantly different from control group. b: significantly different from Fe-NTA treated group. * Means are significantly different at the 0.05 level. $\uparrow$ : Means are significantly different at the 0.01 level. $\$$ : Means are significantly different at the 0.001 level. 
Table (1): Effect of GB and low doses of $\gamma$-irradiation on Kidney function parameters, nitric oxide and lipid peroxide levels in kidneys of Fe-NTA treated groups.

\begin{tabular}{|c|c|c|c|c|}
\hline Group $\quad$ Parameters & $\begin{array}{l}\text { Creatinine } \\
(\mathbf{m g} / \mathbf{d l})\end{array}$ & $\begin{array}{l}\text { BUN } \\
(\mathbf{m g} / \mathbf{d l})\end{array}$ & $\begin{array}{l}\text { NO } \\
\text { (nM/g wet tissue) }\end{array}$ & $\begin{array}{l}\text { LPx } \\
(\mu \mathrm{M} / \mathrm{g} \text { wet tissue) }\end{array}$ \\
\hline Control & $0.36 \pm 0.024^{b \neq}$ & $29.48 \pm 1.12^{b \ddagger}$ & $26.3 \pm 0.57^{b \neq}$ & $181.99 \pm 9.2^{b \neq}$ \\
\hline GB & $0.33 \pm 0.038^{b_{*}^{*}}$ & $23.94 \pm 1.30^{b \ddagger}$ & $28.77 \pm 0.37^{b \neq}$ & $173.98 \pm 13.19^{\mathrm{b}}$ \\
\hline IR & $0.54 \pm 0.019^{a}$ & $47.22 \pm 1.80^{a \ddagger b \dagger}$ & $14.77 \pm 2.34^{a \mathrm{a} b \ddagger}$ & $265.97 \pm 19.06^{a:}$ \\
\hline GB + IR & $0.38 \pm 0.039^{b t}$ & $36.65 \pm 1.75^{b \ddagger}$ & $26.58 \pm 0.13^{b t}$ & $181.82 \pm 5.96^{b \neq}$ \\
\hline Fe-NTA & $0.56 \pm 0.026^{a}$ & $60.79 \pm 2.11^{\mathrm{a} \neq}$ & $16.38 \pm 2.50^{\mathrm{a} \ddagger}$ & $275.31 \pm 26.61^{\mathrm{a}+}$ \\
\hline GB + Fe-NTA & $0.48 \pm 0.011^{\mathrm{a}^{\dagger}}$ & $41.48 \pm 0.78^{\mathrm{a}+\mathrm{b} \ddagger}$ & $27.19 \pm 0.07^{b *}$ & $192.32 \pm 11.35^{\mathrm{b} \ddagger}$ \\
\hline Fe-NTA + IR & $0.6 \pm 0.034^{\text {a: }}$ & $63.76 \pm 7.35$ a & $15.79 \pm 0.32^{\mathrm{a}+}$ & $275.81 \pm 13.75^{\mathrm{a}}$ \\
\hline GB + Fe-NTA + IR & $0.5 \pm 0.038^{a^{\dagger}}$ & $47.12 \pm 2.00^{\mathrm{a} \ddagger \mathrm{b} \dagger}$ & $26.84 \pm 0.15^{b t}$ & $207.82 \pm 3.96^{\mathrm{b}} \dagger$ \\
\hline
\end{tabular}

a: significantly different from control group. b: significantly different from Fe-NTA treated group. * Means are significantly different at the 0.05 level. $\dagger$ : Means are significantly different at the 0.01 level. $\$$ : Means are significantly different at the 0.001 level.

Table (2): Effect of GB and low doses of $\gamma$-irradiation on Kidney antioxidant markers of Fe-NTA treated groups.

\begin{tabular}{|c|c|c|c|c|}
\hline Groups Parameters & $\begin{array}{l}\text { GSH } \\
\text { (mg/g wet tissue) }\end{array}$ & $\begin{array}{l}\text { SOD } \\
\text { (U/g wet tissue) }\end{array}$ & $\begin{array}{l}\text { CAT } \\
(\mu \mathrm{M} / \mathrm{g} \text { wet tissue) }\end{array}$ & $\begin{array}{l}\text { GSH-Px } \\
\text { (Oxidized GSH/min/g } \\
\text { wet tissue) }\end{array}$ \\
\hline Control & $111.74 \pm 0.49^{b \neq}$ & $7.92 \pm 0.92^{\mathrm{b} *}$ & $547.22 \pm 25.42^{\mathrm{b} \ddagger}$ & $0.51 \pm 0.032^{b^{*}}$ \\
\hline GB & $115.31 \pm 3.28^{b \ddagger}$ & $7.25 \pm 0.67^{b:}$ & $587.78 \pm 34.55^{\mathrm{b} \ddagger}$ & $0.52 \pm 0.041^{b^{*}}$ \\
\hline IR & $98.98 \pm 1.16^{a \ddagger b}$ & $5.63 \pm 0.33^{\mathrm{a}^{\dagger}}$ & $466.67 \pm 14.02^{\mathrm{a}^{*}}$ & $0.57 \pm 0.023$ \\
\hline GB + IR & $110.26 \pm 0.38^{b \neq}$ & $7.41 \pm 0.36^{b \ddagger}$ & $506.11 \pm 32.13^{b^{*}}$ & $0.54 \pm 0.012$ \\
\hline Fe-NTA & $69.29 \pm 0.38{ }^{a}$ & $4.13 \pm 0.37^{\mathrm{a}}$ & $421.67 \pm 23.94^{a \neq}$ & $0.59 \pm 0.012^{a^{*}}$ \\
\hline GB + Fe-NTA & $110.81 \pm 0.21$ & $7.42 \pm 0.45^{b \neq}$ & $498.33 \pm 4.00^{b^{*}}$ & $0.55 \pm 0.02$ \\
\hline Fe-NTA + IR & $98.98 \pm 1.16^{a \neq b \neq}$ & $2.65 \pm 0.45^{a t}$ & $417.22 \pm 13.59^{\text {at }}$ & $0.6 \pm 0.008^{\mathrm{a}^{*}}$ \\
\hline GB + Fe-NTA + IR & $110.19 \pm 0.28^{b \ddagger}$ & $5.43 \pm 0.39^{\mathrm{a}^{\dagger}}$ & $460 \pm 31.00^{a^{*}}$ & $0.57 \pm 0.013$ \\
\hline
\end{tabular}

a: significantly different from control group. b: significantly different from Fe-NTA treated group. * Means are significantly different at the 0.05 level. $\dagger$ : Means are significantly different at the 0.01 level. $\$$ : Means are significantly different at the 0.001 level.

\section{Histopathological Studies}

Histological examination of kidney sections derived from normal control rats is shown in Fig. 2A. The circular areas observed in this photograph are the renal Malpighian corpuscle. It is composed of glomerulus surrounded by Bowman's capsule with thin glomerular basement membranes and patent capsular space. Numerous tubules (proximal and distal) lie in the area adjacent to the glomerulus. Treatment of the experimental animals with Ginkgo biloba (GB) represents a no significant change in kidney tissue structure (Fig. 2B). However exposure of the experimental animals to $50 \mathrm{cGy}$ per week for 19 weeks either alone or combined with GB recording some bleeding lesion in the kidney cortical stucture (Figs. 2 C, D). Carcinogenesis of rats by Fe-NTA recording bleeding lesion in all kidney cortical stucture, absence of renal Malpighian corpuscle and accumulation of inflammatory cells (Figs. 2 E, Ea, Eb). Exposure of FeNTA to cGy per week for 19 weeks represents some improvement in renal tissue structure (Fig. 3G). In the meantime, normal renal tissue structure was detected when Fe-NTA (Fig. 3F) or Fe-NTA irradiated groups (Fig. 3H) treated with GB.

\section{Discussion}

Renal cell carcinoma (RCC) is widespread around the world. Phytochemicals have been found to be the most helpful alternative to delay or thwart carcinogenesis. Ginkgo has long been used in Oriental medicine. EGb761, a standard extract of the Ginkgo biloba, is widely used as a medicinal supplement ${ }^{[25]}$, For example, it is used clinically in patients with cognitive function disorders, peripheral blood flow insufficiency, tinnitus, and vertigo ${ }^{[25,26]}$.

Context with the finding of Gupta et al. and Summya et al. ${ }^{[27,28]}$ the kidney of ferric nitrilotriacetate-treated rats showed characteristic morphological changes including bleeding lesion in all kidney cortical stucture, absence of renal Malpighian corpuscle and accumulation of inflammatory cells. 

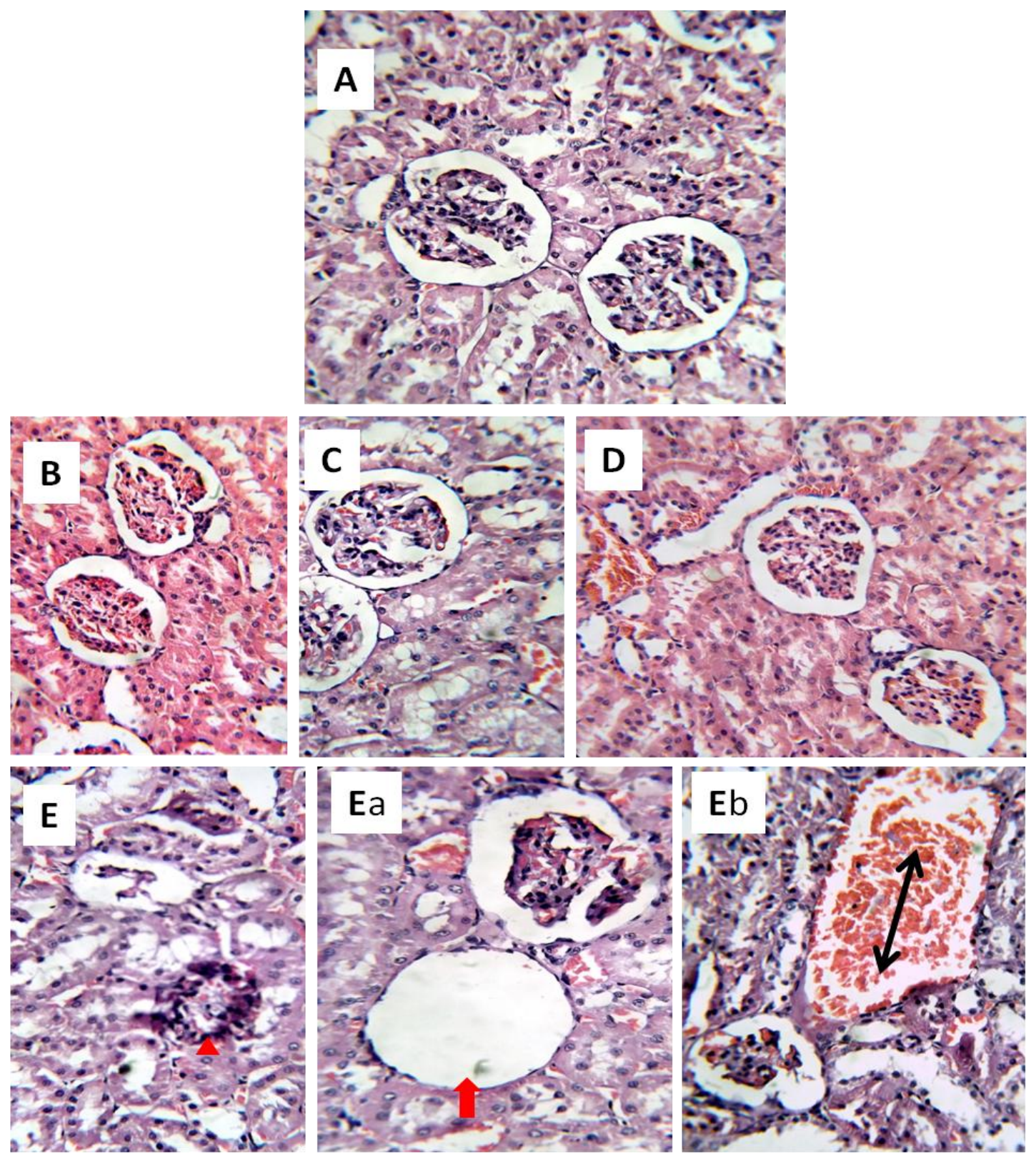

Fig. 2: Photomicrographs of sections in rat kidney stained with H\&E. A: Rat kidney shows cortical part of a control rat. B: GB treated rat kidney show a no significant change. C: Rat kidney exposed to $\gamma$ - radiation shows the appearance of some bleeding lesion. D: GB irradiated rat kidney shows the appearance of some bleeding lesion. E, Ea and Eb: Fe-NTA recording bleeding lesion in all kidney cortical stucture $(\mathfrak{\imath})$, absence of renal Malpighian corpuscle ( $)$ and accumulation of inflammatory cells ( $\mathbf{\Delta}$ ). (All Photomicrographs are at X400).

In the other hand context with the finding of Samir et al [29] the histopathological examination of kidney, $\gamma$ irradiation induced congestion, interstitial haemorrhages and degenerative changes due to ROS production and pro-inflammatory cytokines expression as oxygen species.

Administration of Fe-NTA and iron deposition within kidney tissues which is found to be the main generator of ROS, leading to oxidative damage which further enhances renal lipid peroxidation with concomitant reduction in reduced GSH content and antioxidant enzymes. It induces serum toxicity markers, blood urea nitrogen, creatinine and lactate dehydrogenase ${ }^{[28]}$.
Also, Radiation -induced increases in the levels of free iron in the cell (i.e., iron that is not bound to various proteins), which can promote ROS generation ${ }^{[30]}$. In our cumulative $\gamma$ - irradiation treatments led to a significant depletion in kidney antioxidants markers (GSH, CAT, GSH-px and SOD) when compared with their equivalent values in control rats.Atef et al ${ }^{[31]}$ proposed that GB confers a beneficial radio-preventive effect against irradiation damage induced in rat.In the other hand, we have demonstrated GB inhibits several aspects of $\mathrm{Fe}$ NTA promoted tumor induction response in kidney ${ }^{[32]}$. All the pathological alterations were down regulated by GB pretreatment probable by its modulation of inflammatory mediators and antioxidation ${ }^{[33]}$. 

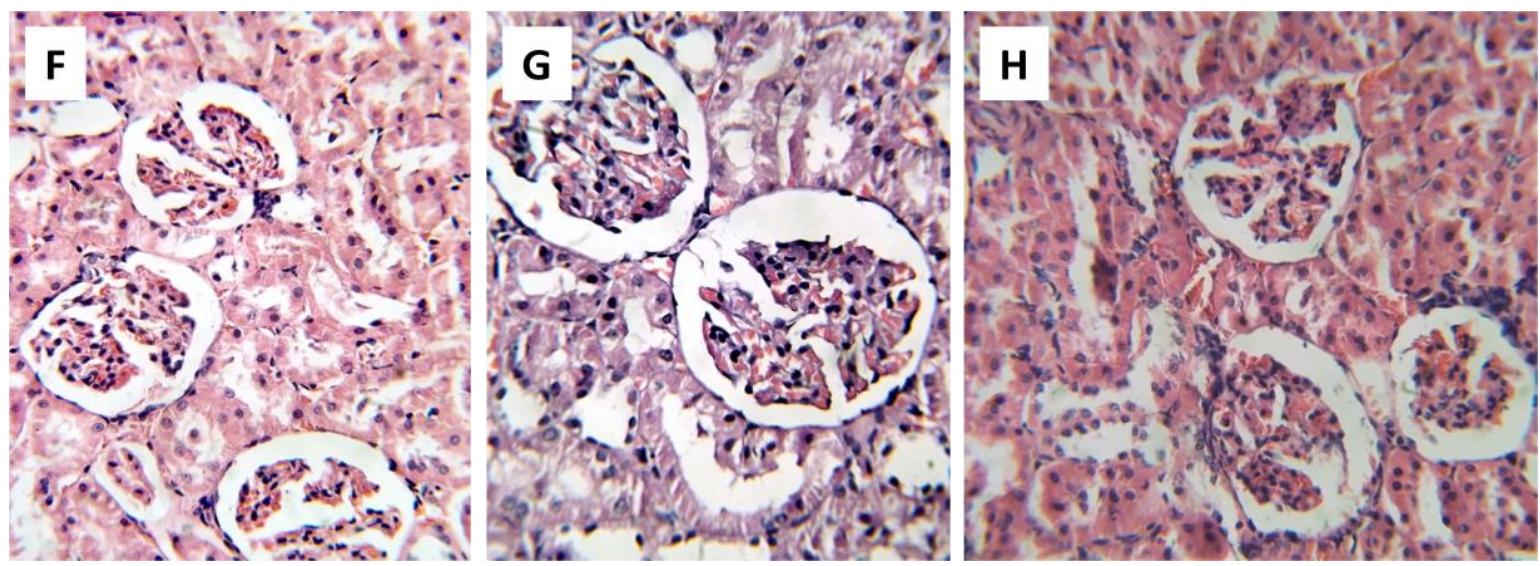

Fig. 3: Photomicrographs of sections in rat kidney stained with H\&E. F: normal renal tissue structure was detected when Fe-NTA group treated with GB. G: Exposure of Fe-NTA to cGy per week for 19 weeks represents some improvement in renal tissue structure. H: Normal renal tissue structure was detected when Fe-NTA irradiated groups treated with GB. (All Photomicrographs are at X400)

In the same direction, it is evident from the present results that prophylactic treatment of GB abrogated GSH and antioxidant armory which was earlier exhausted in scavenging the ROS produced either by irradiation or Fe-NTA treatments. In our study, GB was able to restore SOD, CAT, and GPx levels in kidney tissues. Lin and Chang ${ }^{[34]}$ reported similar elevation in SOD and CAT activities in the epidermis and in the liver of rats after GB treatment. In addition, the depletion of GSH redox cycle seems to be an essential factor that permits lipid peroxidation and ROS accumulation in the Cisplatin treated rats ${ }^{[35]}$. GPx metabolizes $\mathrm{H}_{2} \mathrm{O}_{2}$ to water by using GSH as a hydrogen donor, resulting in the generation of GSSG. GB was able to decrease GSSG/GSH ratio, probably by reversing the depletion of GSH concentration.

There was concomitant increase in plasma toxicity markers like BUN and creatinine in Fe-NTA treated group as compared to control. Our results are in good agreement with those previously reported ${ }^{[6]}$. On the contrary, lower BUN and creatinine levels were found in the rats administered with GB as compared to Fe-NTA treated group. Hence the decrease in the levels of these enzymes and attenuation of antioxidant armory may be possibly by nephroprotective efficacy of GB ${ }^{[36]}$.

In our research, the NO levels was decreased following Fe-NTA administration context with the finding of Gupta et al. ${ }^{[37]}$ home proposed Fe-NTA administration markedly increased the BUN and serum creatinine level which was coupled with a marked lipid peroxidation, reduced activity of glutathione and decreased total nitric oxide levels of rat kidneys coupled with significant morphological alterations. Many researches has shown that the catalytic activity of iNOS is enhanced by excess non-haem iron that causes the release of nitric oxide which combines with already present superoxide anion to form a toxic mediator 'peroxynitrite' and results in cellular membrane damage by lipid peroxidation and oxidation ${ }^{[38]}$. GB inhibited inflammatory iNOS expressions in LLC-PL1 cells, the classic target of anti- inflammatory pathways. However, Gulec et al. [39] previously reported that GB protected against Fe-NTA induced nephrotoxicity by suppressing adenosine deaminase activities, and GB showed a tendency to inhibit NO levels but with no statistical significance. Although not all the results are uniform, an effect on iNOS by GB seems reasonable and accurate.

Context with finding of Mei et al. ${ }^{[40]}$, a significant decrease in levels of kidney caspase 3 was observed in group subjected to Fe-NTA. Caspase 3 seems to play an important role in Fe-NTA-dependent loss of cell viability because a relatively specific peptide inhibitor of caspase 3. Fe-NTA induced notable ROS, which played a protective role in apoptosis by inhibiting Caspase-3 activation. Fe-NTA-induced ROS increased mRNA and protein level of anti-apoptosis $\mathrm{Bcl}-2$ and decreased mRNA protein level of pro-apoptosis gene Bax, As a result, maintaining mitochondrial membrane potential ${ }^{[40]}$. The prophylactic and therapeutic treatment with GB significantly increases caspase 3 level due to its inhibition to ROS ${ }^{[41]}$.

In this study, GB only caused a modest reduction in caspase-3 expression in vivo. This might reflect the incomplete suppression of caspase cascade by GB. Accordingly Luo et al. ${ }^{[42]}$ revealed that GB protected against neuron cell death with only a minor effect on caspase-3 expression. ROS production can be boosted by inducing mitochondrial dysfunction and caspase cascade activation via the disrupted respiratory chain or by depleting and inactivating glutathione and thus shifting the cellular redox status. Our research suggested that caspase cascade was not a major pathway for GB's protection against Fe-NTA renal carcinogenesis.

From the previous discussed results it could be concluded that GB associated with upregulation of antioxidant armory, down regulate plasma toxicity markers, down regulate caspase 3 and normalize renal tissue structure in rats affected by Fe-NTA and / or gamma irradiation. 


\section{References}

1) Suzy, V. T. and Frank, M. T. (2013). Iron and cancer: more ore to be mined. Nat Rev Cancer, 13(5):342-355.

2) Inous, S. and Kawanishi, S. (1987). Hydroxyl radical production and human DNA damage induced by ferric nitrilotriacetate and hydrogen peroxide. Cancer Res, 47:6522 - 6527.

3) Jemal, A., Siegel, R., Xu, J. and Ward, E. (2010). Cancer statistics. A Cancer Journal for Clinicians, 60(277): 300 - 304.

4) Awai, M., Narasaki, M., Yamanoi, Y. and Seno, S. (1979). Induction of diabetes in animals by parenteral administration of ferric nitrilotriacetate. A model of experimental hemochromatosis. Am.J.Pathl., 95:663673.

5) Hamazaki, S., Okada, S., Ebina, Y. and Midorikawa, O. (1985). Acute renal failure and glycosuria induced by ferric nitrilotriacetate in rats. Toxicol. Appl. Pharmacol., 77:267-274.

6) Rehman, U. M. and Sultana, S. (2011). Attenuation of oxidative stress, inflammation and early markers of tumor promotion by caffeic acid in Fe-NTA exposed kidneys of Wister rats. Mol Cell Biochem, 357:115124.

7) Liu, M., Okada, S. and Kawabata, T. (1993). Radical promoting free iron level in serum of rats treated with other iron chelate complexes. Acta Med Okayama, 45:401-408.

8) Zhang, D., Okada, S., Kawabata, T. and Yasuda, T. (1995). An improved simple colorimetric method for the quantization of nonbound iron in serum.Biochem. MolBiolInt, 35:635-641.

9) Samuelson, G. (1992). Drugs of Natural Origin.Swedish Pharmaceutical Press, Stockhlm, p. 86.

10) Pincemail, J., Dupuis, M., Nasr, C., HaagBerrurier, M., Anton, R. and Deby, C. (1989). Superoxide anion scavenging effect and superoxide dismutase activity of Ginkgo Biloba extract. Experientia, 45:708-712.

11) Akiba, S., Kawauchi, T., Oka, T.and Sato, T. (1998). Inhibitory effect of the leaf extract of Ginkgo biloba L. on oxidative stress-induced platelet aggregation. Biochem Mol BiolInt, 46:1243-1248.

12) Akisu, M., Kultursay, N., Coler, I. and Huseyinov, A. (1998). Platelet-activating factor is an important mediator in hypoxic ischemia brain injury in the newborn rat. Flunarizine and Ginkgo biloba extract reduce PAF concentration in the brain. Biol Neonate, 74:439-444.

13) Kleijnen, J. and Knipschild, P. (1992). Ginkgo biloba.Lancet, 340:1136-1139.

14) Kamat, J. P., Boloor, K. K., Devasagayam, T. P. A. and Venkatachalam, S.R. (2000). Antioxidant properties of Asparagus racemosus against damage induced by $\gamma$-radiation in rat liver mitochondria. $J$ Ethnopharmacol, 71:425-435.
15) Breen, A. P. and Murphy, J. A. (1995). Reactions of oxyl radicals with DNA. Free Radical Biol Med, 18:1033-1077.

16) Jagetia, G. C., Rajanikant, G. K., Shaival, K., Rao, M. and Baliga, S. (2003). Alteration in the glutathione, glutathione peroxidase, superoxide dismutase and lipid peroxidation by ascorbic acid in the skin of mice exposed to fractionated $\gamma$ radiation. Clin Chim Acta, 332:111-121.

17) Fang, Y., Yang, S. and Wu, G. (2002). Free radicals, antioxidants, and nutrition. Nutrition, 18:879-887.

18) Lui, X., Dong, M., Chen, X., Jiag, M., Lv, X. and Yan, G. (2007). Antioxidants activity and phenolics of an endophytic xylaria Sp. from Ginko biloba. Food chemistry, 105(2):548-554.

19) Beutler, E., Duron, O. and Kelly, B.M. (1963). Improved method for the determination of blood glutathione. J Lab Clin Med. 61:882 -888.

20) Gross, R.T., Bracci, R., Rudolph, N., Schroeder, E. and Kochen, J.A. (1967). Hydrogen peroxide toxicity and detoxification ion in the erythrocytes of newborn infants. Blood, 29:337-342.

21) Minami, M. and Yoshikawa, H. (1979). A simplified assay method of superoxide dismutase. Clinica Chimica Acta. 92:337.

22) Sinha, A. K. (1972). Colorimetric assay of catalase. Anal. Biochem. 47:389-394.

23) Yoshioka, T. K., Kawada, T., Shimada, M. and Mori, M. (1979). Lipid peroxidation in maternal and cord blood and protective mechanism against activated-oxygen toxicity in the blood. Am J Obstet. Gynecol, 135:372-376.

24) Miranda, K.M., Espey, M.G. and Wink, D.A. (2001). A rapid, simple spectrophotometric method for simultaneous detection of nitrate and nitrite. Nitric Oxide, 5:62-71.

25) Jacobs, B. P. and Browner, W. S. (2000). Ginkgo biloba: a living fossil. Am J Med, 108:341-342.

26) De Feudis, F. V. (1991). Coronary atherosclerosis: current therapeutic approaches and future trends. Life Sci, 49:689-705.

27) Gupta, A., Sharma, S., Kaur, I. and Chopra, K. (2009). Renoprotective Effects of Sesamol in Ferric Nitrilotriacetate-Induced Oxidative Renal Injury in Rats. Basic \& Clinical Pharmacology \& Toxicology, 104:316-321.

28) Summya, R., Nemat, A., Sana, N., Syed, K. H. and Sarwat, S. (2013). Amelioration of Renal Carcinogenesis by Bee Propolis: A Chemo Preventive Approach. ToxicolInt, 20(3):227-234.

29) Samir, A. E., Mohamed, M., Ahmed, H. E., Ahmed, H. E. and Zein, S. I. (2012). Protective Effect of L -carnitineAgainst $\gamma$-Rays Irradiationinduced Tissue Damage in Mice. American J Biochem Mol Biol, 2:120-132.

30) Halliwell, B. (1999). Antioxidant defence mechanisms: From the beginning to the end. Free Radical Research, 31:261-272. 
31) Atef, A. F., Mohamed, El-Sh., Ahmed, H., Gamal, A., Magda, H. B. And Ashraf, M. B. (2009). Ameliorative effect of ginkgo biloba extract on gamma radiation injury: histological and histochemical evaluation in rats. Mansoura $J$ Forensic Med Clin Toxicol, XVII(2).

32) Iqbal, M., Giri, U., Giri, D. K., Alam, M. S. and Athar, M. (1999). Age-dependent renal accumulation of 4-hydroxy-2-nonenal (HNE)modified proteins following parenteral administration of ferric nitrilotriacetate commensurate with its differential toxicity: Implications for the involvement of HNE-protein adducts in oxidative stress and carcinogenesis. Arch BiochemBiophys, 365:101-112.

33) Yan-Hong, Z., Jie-Ping, Y., Yi-Fei, L. ,Xiao-Jun T., Ming, M. and Peng, L. V. (2006). Effects of Ginkgo bilobaExtract on Inflammatory Mediators (SOD, MDA, TNF- $a$, NF- $\kappa$ Bp65, IL-6) in TNBSInduced Colitis in Rats. Hindawi Publishing Corporation Mediators of Inflammation, 1-9.

34) Lin, S. Y. and Chang, H. P. (1997). Induction of superoxide dismutase and catalase activity in different rat tissues and protection from UVB irradiation after topical application of Ginkgo biloba extracts. Methods and Findings in Experimental and Clinical Pharmacology, 19(6):367-371.

35) Atasayar, S., Gurer-Orhan, H., Orhan, H., Urel, B. G., Girgin, G. and Unes, H. O. (2009). Preventive effect of aminoguanidine compared to vitamin $\mathrm{E}$ and $\mathrm{C}$ on cisplatin-induced nephrotoxicity in rats. Experimental and Toxicologic Pathology, 61(1):23-32.

36) Newairy, A. S., Salama, A. F., Hussien, H. M. and Yousef, M. I. (2009). Propolis alleviates aluminium- induced lipid peroxidation and biochemical parameters in male rats. Food Chem Toxicol, 47: 1093-1098.

37) Gupta, A., Sharma, S. and Chopra, K. (2008). Reversal of iron-induced nephrotoxicity in rats by molsidomine, a nitric oxide donor. Food Chem Toxicol, 46(2):537-543.

38) Chen, L., Wang, Y., Kairaitis, L. K., Zhang, B. H. and Harris, D. C. (2001). Molecular mechanisms by which iron induces nitric oxide synthesis in cultured proximal tubule cells. Exp Nephrol, 9:198-204.

39) Gulec, M., Iraz, M., Yilmaz, H. R., Ozyurt, H. and Temel, I. (2006). The effects of Ginkgo biloba extract on tissue adenosine deaminase, xanthine oxidase, myeloperoxidase, malondialdehyde, and nitric oxide in cisplatin-induced nephrotoxicity. Toxicology and Industrial Health, 22(3):125-130.

40) Mei, L., Shu-Jie, L., Yong-Ning, X., Shu-Sheng, J., Rui-Jin, X. and Shi-Ying, X. (2015). Ferric nitrilotriacetate (Fe-NTA)-induced reactive oxidative species protects human hepatic stellate cells from apoptosis by regulating Bcl-2 family proteins and mitochondrial membrane potential. Int J Clin Exp Med, 8(10):18074-18081.

41) Song, J., Liu, D., Feng, L., Zhang, Z., Jia, X. and Xiao, W. (2013). Protective Effect of Standardized Extract of Ginkgo biloba against Cisplatin-Induced Nephrotoxicity. Evidence-Based Complementary and Alternative Medicine, 2013:1- 11.

42) Luo, Y., Smith, J. V. and Paramasivametal, V. (2002). Inhibition of amyloid- $\beta$ aggregation and caspase- 3 activation by the Ginkgo biloba extract EGb761. Proceedings of the National Academy of Sciences, 99(19):12197-12202. 